\section{Creating a buzz}

This year's BDIA Dental Showcase 2018 set the perfect stage for EKC to create a buzz, with the professional team's very own 'queen bee' attracting many visitors.

EKC says it was proud to support the Bumblebee Conservation Trust in memory of a beloved friend, Maria Ricketts, who worked as a Production Manager at The Dentist and Dental Update for more than 20 years before she lost her battle to cancer.

Delegates and exhibitors made a beeline for EKC's stand where they were encouraged to donate some of their loose change to a good cause.

EKC would like to thank everyone at the BDIA Dental Showcase who contributed to the cause. EKC donated a grand total of $£ 373.85$ to the Bumblebee Conservation Trust, which will be put to good use in ensuring that Britain's remaining bumblebee populations remain integral to our biodiversity.

For more information visit www.bumblebeeconservation.org or www.ekcommunications.co.uk

\section{Remain competitive}

Although dental implants are a now a widely accepted treatment modality for many patients in Britain, some practitioners lack the time, facilities or inclination to train in implantology.

To avoid losing patients to competitors who are qualified to place implants, Ten Dental is offering a solution through its own dedicated Implant Restoration Course (IRC).

Highly knowledgeable implant surgeons - Drs Martin Wanendeya and Nik Sisodia - combine years of experience to teach the essential skills required to restore implants.

Practitioners are able to diagnose and plan treatment, then restore the implants once the surgery has been performed by an implantologist. This allows you to retain your patient base by remaining a trusted and integral part of the implant treatment journey.

For practitioners who aspire further, Ten Dental's IRC can act as a solid foundation from which to develop their skills in the future - opening a new avenue into the lucrative implant market.

More information about Ten Dental and The Implant Restoration Course is available by visiting Facebook/Implant Restoration Course-IRC, emailing courses@tendental.com, visiting www.implant-restoration.com or calling 02076227610.

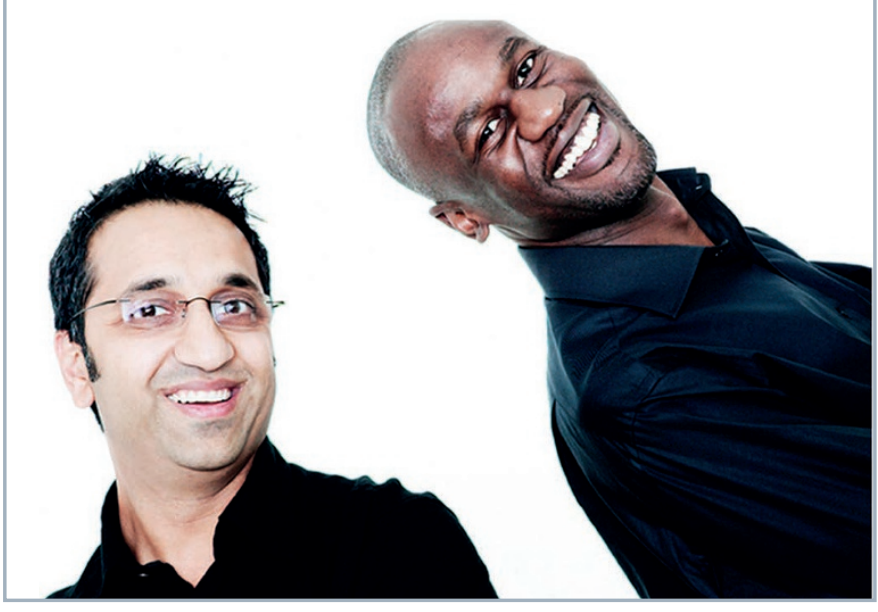

\section{Time-saving and easy to use bonding}

Tokuyama's Universal Bond produces high bond strengths to almost all dental substrates and does so without having to add activators or primers. There is also no need to wait after placement as it self-cures.

Universal Bond adheres to dentition on contact and then self-polymerises, says the company, and overall, it reduces time with the curing lamp.

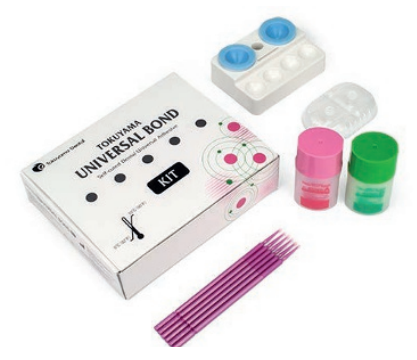

Simply mix one drop of Bond A and Bond B together to trigger the chemical cure sequence and this provides worry-free polymerisation where curing lights cannot reach.

The product will bond any two interfaces such as porcelain, metal, zirconium and acrylic with no primers required and because of its high adhesive performance, Tokuyama says it is the only real universal bonding agent for all materials. It can also be used with any competitor bondable filling and cement with universal applicability.

More information is available at www.trycare.co.uk/universalbond or by calling 01274885544 .

\section{Software boost for dental automated recalls}

Dentally, the UK's leading cloud dental software, has unveiled a new product development for automated recalls to improve the process around notifying patients that they are due for a recall appointment.

The new development means that users can set up recall messaging workflows within the software, which will automate the process to send the patient an email, SMS or letter at the appropriate and correct time.

Users can customise the way they set up the recall workflows based on the recall type, so for example, for the dentist or hygienist, as well as ensuring it is the right one based on payment plan, practitioner, recall date and type of correspondence.

The messaging can include a link to dentr - an online booking app that has been designed for Dentally - so patients can book their recall online.

James Harker, CTO and Co-Founder, said: 'It has been designed in an easy to use and intuitive way, so it runs seamlessly from the patient's perspective and it saves valuable staff time and doesn't overwhelm the day-to-day running of the practice.'

More information is available at https://dentally.co/ or email hello@dentally.co or call 02038565610. 